\title{
New Cosmological Model and Observational Data Interpretation
}

\section{Branislav Vlahovic ${ }^{\mathbf{1}}$}

North Carolina Central University

Fayetteville st. 1801, Durham, North Carolina 27707, U.S.A.

E-mail: vlahovic@nccu.edu

\section{Maxim Eingorn}

North Carolina Central University

Fayetteville st. 1801, Durham, North Carolina 27707, U.S.A.

E-mail: maxim.eingornegmail.com

The paradigm of $\Lambda C D M$ cosmology works impressively well and with the concept of inflation it explains the Universe evolution. However, there are still a few concerns; after much effort there is no detection of dark matter and there are significant problems in the theoretical description of dark energy. Within FLRW formalism we consider a variant of the cosmological model with the spherical space filled with an additional perfect fluid with the constant negative parameter $-1 / 3$ in the linear equation of state. This fluid may be called quintessence or a frustrated network of cosmic strings. We compare our model with the standard concordance one and show that it satisfies the supernovae data. However, some data may require new interpretation, e.g., the CMB data. We discuss a possibility that the inflation scenario may completely lose its necessity.

The European Physical Society Conference on High Energy Physics

18-24 July, 2013

Stockholm, Sweden

1

Speaker 


\section{Introduction}

Inspired by [1], in this brief paper we answer the following question: "Is there a possibility from the mathematical point of view that the last scattering surface is approximately point-like in the closed FLRW Universe?" In other words, is inflation really necessary in the case of the spherical space? Obviously, if we observe CMB photons which originate from a rather small causally connected spatial region, then the famous horizon problem is easily resolved in the elegant geometric way. In what follows we consider the corresponding standard metric:

$$
d s^{2}=c^{2} d t^{2}-a^{2}(t)\left[d \chi^{2}+\sin ^{2} \chi\left(d \theta^{2}+\sin ^{2} \theta d \psi^{2}\right)\right],
$$

with hyperspherical coordinates $\chi \in[0, \pi] ; \theta \in[0, \pi] ; \psi \in[0,2 \pi)$.

\section{The pure $\Lambda$ CDM model}

In the framework of the concordance model the Hubble parameter squared reads

$$
H^{2}=\left(\frac{\dot{a}}{a}\right)^{2}=\frac{\kappa \bar{\rho} c^{4}}{3 a^{3}}+\frac{\Lambda c^{2}}{3}-\frac{c^{2}}{a^{2}}=H_{0}^{2}\left(\Omega_{M} \frac{a_{0}^{3}}{a^{3}}+\Omega_{\Lambda}+\Omega_{K} \frac{a_{0}^{2}}{a^{2}}\right),
$$

where the following standard designations are introduced:

$$
\Omega_{M}=\frac{\kappa \bar{\rho} c^{4}}{3 H_{0}^{2} a_{0}^{3}}, \quad \Omega_{\Lambda}=\frac{\Lambda c^{2}}{3 H_{0}^{2}}, \quad \Omega_{K}=-\frac{c^{2}}{a_{0}^{2} H_{0}^{2}}, \quad \kappa=\frac{8 \pi G_{N}}{c^{4}} .
$$

With the help of the values $0.31,0.69$ and -0.001 [2] for $\Omega_{M}, \Omega_{\Lambda}$ and $\Omega_{K}$, respectively, we obtain for the deceleration parameter value 0.535

$$
-q=\frac{1}{a H^{2}} \frac{d^{2} a}{d t^{2}}=\left(\frac{H_{0}}{H}\right)^{2}\left\{-\frac{\Omega_{M}}{2}\left(\frac{a_{0}}{a}\right)^{3}+\Omega_{\Lambda}\right\} .
$$

Introducing dimensionless quantities $\tilde{a}=a / a_{0}, \tilde{t}=H_{0} t, \tilde{a}(0)=1, \tilde{a}\left(-\tilde{t}_{0}\right)=0$, for the age of the Universe we obtain:

$$
\tilde{t}_{0}=\int_{0}^{1} \frac{\sqrt{\tilde{a}} d \tilde{a}}{\sqrt{\Omega_{M}+\Omega_{K} \tilde{a}+\Omega_{\Lambda} \tilde{a}^{3}}}=0.96,
$$

which corresponds to 13.9 billions of years (because $H_{0} \approx 2.2 \times 10^{-18} \mathrm{~s}^{-1}$ [2]).

Let us write down the approximate condition of light traveling between the antipodal points during the age of the Universe:

$$
\int_{-t_{0}}^{0} \frac{c d t}{a(t)}=\pi, \quad \sqrt{-\Omega_{K}} \int_{0}^{1} \frac{d \tilde{a}}{\sqrt{\Omega_{M} \tilde{a}+\Omega_{K} \tilde{a}^{2}+\Omega_{\Lambda} \tilde{a}^{4}}} \approx 0.1 \neq \pi .
$$

Thus, this condition is not satisfied in the concordance model.

\section{Quintessence or a frustrated network of cosmic strings}

Let us supplement the commonly accepted Universe composition with an additional perfect fluid, with the constant parameter $-1 / 3$ in the linear equation of state (this fluid may be 
called either quintessence or a frustrated network of cosmic strings and plays an important role for deriving reasonable gravitational potentials of inhomogeneities in the discrete theory of scalar cosmological perturbations [3, 4]). Then instead of (2) we have

$$
H^{2}=\left(\frac{\dot{a}}{a}\right)^{2}=H_{0}^{2}\left(\Omega_{M} \frac{a_{0}^{3}}{a^{3}}+\Omega_{\Lambda}+\Omega_{Q} \frac{a_{0}^{2}}{a^{2}}+\Omega_{K} \frac{a_{0}^{2}}{a^{2}}\right) .
$$

The parameters of the model may be defined from the evident conditions

$$
\Omega_{M}+\Omega_{\Lambda}+\Omega_{Q}+\Omega_{K}=1, \quad-\frac{\Omega_{M}}{2}+\Omega_{\Lambda}=-q=0.535
$$

and the additional condition of light traveling between the antipodal points

$$
\sqrt{-\Omega_{K}} \int_{0}^{1} \frac{d \tilde{a}}{\sqrt{\Omega_{M} \tilde{a}+\Omega_{Q} \tilde{a}^{2}+\Omega_{K} \tilde{a}^{2}+\Omega_{\Lambda} \tilde{a}^{4}}}=\pi .
$$

They all can be satisfied, e.g., by the following set of values: $\Omega_{M}=0.040, \Omega_{\Lambda}=0.555$, $\Omega_{Q}=0.721, \Omega_{K}=-0.316$. This choice is also interesting in view of the fact that there is no dark matter: the baryonic contribution may be sufficient. Besides, the made choice satisfies the supernovae data approximately with the same accuracy as the $\Lambda \mathrm{CDM}$ model. Of course, all other cosmological tests require additional investigation lying beyond the scope of this short paper.

\section{Conclusion}

In the $\Lambda \mathrm{CDM}$ model supplemented with the perfect fluid with the constant negative parameter $-1 / 3$ in the linear equation of state in the spherical space there is an elegant solution of the horizon problem without inflation: under the proper choice of the parameters light travels between the antipodal points during the age of the Universe. In the constructed model the gravitational potential of each mass is convergent (see [4]). Our model satisfies the supernovae data and may represent a promising alternative to modern inflationary theories.

\section{Acknowledgements}

We acknowledge support by NSF CREST award HRD-0833184 and NASA grant NNX09AV07A.

\section{References}

[1] B. Vlahovic, New Cosmological Model and Its Implications on Observational Data Interpretation [physics/1303.3203].

[2] Planck 2013 results. XVI. Cosmological parameters, Planck Collaboration (2013) [astro$\mathrm{ph} / 1303.5076]$.

[3] M. Eingorn and A. Zhuk, Hubble flows and gravitational potentials in observable Universe, JCAP 09 (2012) 026 [astro-ph/1205.2384].

[4] A. Burgazli, M. Eingorn and A. Zhuk, Can quintessence and phantom cause the late time acceleration of the Universe?, submitted to JCAP (2013) [astro-ph/1301.0418]. 